\title{
UNA PROPUESTA DE ORGANIZACIÓN BASADA EN LA TEORÍA DE SISTEMAS
}

Juan Cevallos A.*

\section{RESUMEN}

El presente artículo trata sobre el análisis de sistemas, orientado hacia la solución de problemas complejos que se presentan en las organizaciones, a fin de planificar el diseño operativo de la organización, en especial, el sistema de trabajo de alto desempeño.

Palabras Clave: Sistema, método, congruencia, grupo estratégico.

\section{ABSTRACT}

The present article is about the analysis of systems for the solution of complex problems that presents in the organizations in order to plan the operative design of the organization especially the system of work of high level.

Words Key: System, method, consistency, strategic group

\section{INTRODUCCIÓN}

En el mundo moderno la arquitectura de la organización busca convertirse en una ventaja competitiva, para ello se toma en cuenta aspectos como la innovación tecnológica, la orientación al cliente, la oferta de productos y servicios a bajo costo y la habilidad para mantener esas competencias en el largo plazo y otros relacionados. Esto implica para todo directivo de una organización: comprendery aprender las técnicas relacionadas con el diseño de su organización el cual para ser eficaz es un proceso inacabable.

Para tener un adecuado sistema organizacional es necesario ir más allá de lo tradicional. Lo tradicional radica en considerar sólo: los presupuestos, los balances generales, los cuadros de personal, los organigramas, las relaciones entre personas, el trabajo, las estructuras formales y las prácticas y el comportamiento informal. Para ello es necesario considerar la organización como un sistema altamente integrado. Se requiere entender la organización. La organización es un sistema cuyo desempeño está determinado por el grado de alineación entre sus principales componentes; se busca la arquitectura adecuada, para lograr la alineación perfecta.

Para una adecuada arquitectura hay que tomar en cuenta lo estructural y lo social, con la finalidad de obtener un modelo conceptual de la organización.

\footnotetext{
* Magister en Ingeniería Industrial, Ingeniero en Industrias Alimentarias. Instituto de Investigación. Facultad de Ingenie ría Industrial. UNMSM

E-mail : d260003@unmsm.edu.pe
}

Las organizaciones muestran las características de cualquier sistema básico, así tenemos:
a. La interrrelación interna.
b. La capacidad de interrelación.
c. El equilibrio.
d. La adaptación.

Es decir todo ello se basa en la teoría de los sistema abiertos, lo cual es distinto al viejo esbozo de la estructura piramidal.

\section{SOBRE LA TEORÍA SISTÉMICA DE LA ORGANIZACIÓN}

\section{Definición de Sistema}

Es un método para conocer mejor la organización. Implica estudiar la organización con base al análisis y sintesis de sistemas, la determinación de funciones que se presentan en la organización y la determinación de los cambios en el tiempo.

La definición clásica de Sistema, fue dada por Bertalanffy (1968) (fundador de la Teoría General de Sistemas): "Un sistema puede definirse como un complejo de elementos $\mathrm{f} 1, \mathrm{f} 2, \ldots, \mathrm{fn}$, en interacción."

\section{Conceptos Basicos Sobre Sistemas}

a. Las estructuras de los sistemas que crea el hombre pueden tomar como base el funcionamiento de los sistemas naturales.

b. El todo es diferente que la suma de sus partes. El todo puede ser mayor o menor que. 
c. Los sistemas que desarrolla el hombre deben tomar en cuenta las culturas y los intereses cambiantes de los grupos humanos. Se debe apreciar con una concepción global.

d. Los sistemas complejos tienden a conservarse mediante intercambios con el medio y la actuación de dispositivos de regulación; y asimismo, tienden a crecer y desarrollarse. Sin embargo, el crecimiento de los subsistemas se da a diferentes ritmos.

e. Todo sistema tiene un orden, que es complejo y está organizado.

f. Los sistemas partiendo de situaciones diferentes pueden llegar a estados iguales; $y$ asimismo, partiendo de situaciones iguales pueden llegar a estados diferentes.

g. Los sistemas complejos aprenden y se adaptan ante cambios acaecidos en el propio sistema o en su entorno.

h. Los diversos sistemas y subsistemas se complementan entre sí de manera dinámica en el tiempo.

i. No es posible obtener un conocimiento total de ningún sistema, y por tanto siempre habrá incertidumbre sobre el futuro rumbo que tome un sistema.

j. Muchas causas explican las relaciones inter e intrasistémicas. Identificar el mayor número de estímulos externos e internos es importante a fin de reconocer los factores más significativos.

k. Existe una gran interdependencia entre los sistemas; todo sistema depende en gran medida de otros sistemas.

I. Todos los sistemas interactúan produciéndose reacciones en cadena. Se busca aumentar los efectos positivos de la reacción en cadena y disminuir los efectos negativos.

m. La actuación de cada subsistema se difunde por el sistema entero, con distinta intensidad.

n. Todo sistema es complejo desde el punto de vista conceptual y está compuesto de estructuras reales.

ñ. Los sistems humanos se caracterizan por una complejidad creciente de: conocimientos, organización, relaciones y funciones, entornos, tecnologías, necesidades de información.

o. Hay diversos niveles de complejidad que pasan desde el subatómico, atómico, partícula coloidal, célula, hasta llegar al ser humano, y a entidades sociales de aspecto multiforme como: educativas, económicas, militares, ideológicas, literarias, artísticas, políticas, etc.

\section{MÉTODOS Y TÉCNICAS DE SISTEMAS}

\section{EI Método Sistémico Integrado}

Es un método que toma en cuenta los hechos del exterior, con base a ello desarrolla hipótesis y aplica los métodos inductivo y deductivo para experimentar y con base a los resultados de la experimentación, planifica futuros cambios o mejoras.

El análisis de sistemas es un método para comprender la estructura, funciones y relaciones de los sistemas. El análisis de sistemas es aplicable a toda situación en la que hayan de adoptarse decisiones de amplio alcance y se disponga de tiempo suficiente para estudiar la situación.

En los procesos de análisis de sistemas se utilizan experiencias, conocimientos, técnicas y procedimientos que proceden en gran parte de la práctica militar, empresarial e industrial, y de las teorías elaboradas por pensadores, científicos y técnicos a lo largo de diversas épocas y para hacer frente a diversas situaciones.

El análisis de sistemas es una actividad multidisciplinaria orientada hacia la solución de problemas, que responde a la necesidad de manejar las complejas situaciones que se presentan actualmente en las organizaciones y en las empresas públicas y privadas.

En el análisis de sistemas hay ciertas características comunes que se presentan cuando se aplica a las diversas organizaciones, ellas son:

a. Contexto: Implica considerar la empresa y su contexto, es decir su entorno y la sociedad.

b. Métodos: Que se entiende como una síntesis de comprensión, invención, análisis, diseño, intuición, juicio y concepción científica.

c. Instrumentos: Pueden ser la lógica, estadística, matemáticas, tecnología y las ciencias utilizadas por grupos multidiscipli-narios.

d. Objetivo: Determina las respuestas que esperan obtener.

e. Clientes: Personas o entidades que tienen interés en obtener las respuestas precisadas en los objetivos, por lo general están relacionadas con problemas o retos por avanzar. 
f. Relación: Interacción continua entre el grupo de análisis y los clientes a lo largo del trabajo.

Actividades para hacer Análisis de Sistemas

a. Reunir datos relativos al problema, a la experiencia y al conocimiento científico relacionado, y si es preciso buscar nuevos datos y elaborar nuevos conocimientos.

b. Examinar los objetivos sociales relacionados con el problema.

c. Explotar diversas opciones para cumplir los objetivos y diseñar o inventar nuevas posibilidades.

d. Reconsiderar el problema y su posible reformulación a la luz de los conocimientos acumulados durante el análisis.

e. Evaluar el impacto de las diversas formas de actuación.

f. Comparar las opciones, aplicando diversos criterios a sus consecuencias.

g. Presentar los resultados del estudio, de forma que facilite la elección.

h. Contribuir a llevar a cabo las actuaciones elegidas.

i. Evaluar los resultados

Tipos de Análisis.

Los tipos de análisis podrían clasificarse en:

a. Estructural: Que considera los componentes y elementos de la estructura del sistema. Tiene un carácter más bien estático.

b. Funcional: Permite definir los flujos de perso nas, materiales, dinero, energías u otras corrientes de masa - energía que transcurre por los diversos circuitos de la organización.

c. Relacional: Permite determinar los elementos, las partes componentes o subsistemas de la organización que se hallan íntimamente interrelacionados, pudiendo aislarse y definirse hasta cierto punto sus circuitos y otros muchos tipos de conexiones.

Los tres tipos de análisis están a su vez interrelacionados.

\section{Esquemas para el análisis de sistemas}

Uno de los más sencillos es el que considera los siguientes elementos:
- Tipo del sistema

- Determinación de objetivos

- Estructura

- Funciones

- Entornos

- Relaciones

- Resumen de la situación presente

- Líneas de acción para alcanzar objetivos y metas.

- Determinación de metas secuenciales.

Cronograma

- Propuesta de nuevos objetivos.

- Escenarios o modelos prospectivos a corto, me diano y largo plazo.

- Análisis de los puntos débiles y fuertes.

- Análisis de las amenazas y oportunidades del entorno.

- Análisis de costos.

- Análisis de los medios de realización: humanos, naturales, tecnológicos, financieros, etc.

El análisis puede alcanzar mayores detalles y considerar:

- Objetivos concretos.

- Estrategia y táctica

- Evaluación de actividades

- Puntos fuertes y débiles

- Análisis del entorno.

- Análisis de los sistemas de comunicación e información.

\section{MODELO DE TRABAJO DE LA INGENIERIA DE SISTEMAS}

Arbones, en su texto Ingeniería de Sistemas, plantea el siguiente modelo de trabajo:

Etapas:

$$
\begin{aligned}
& \text {.Planeación, } \\
& \text {.Desarrollo } \\
& \text { Acción. }
\end{aligned}
$$

Las Fases de Planeación y Desarrollo son: Estudio de sistemas, Planeación del Proyecto y Planeación del Desarrollo. La Planeación del Desarrollo comprende: Determinación del problema, Selección de los Objetivos, Síntesis del Sistema, Análisis del Sistema, Selección del Mejor Sistema, Comunicación de los Resultados.

Las Fases de la Acción son: Estudios durante el desarrollo y Prosecución Técnica.

Una parte fundamental en todo el proceso es la Construcción del Modelo del Sistema, que debe elaborarse luego de la Fase de Planeación y Desarrollo. Para la propuesta de modelo tienen que tenerse presentes los principios y características de los sistemas, lo que se verá a continuación. 


\section{PRINCIPIOS Y CARACTERISTICAS DE LOS SISTEMAS}

\section{PRINCIPIOS:}

a. Subsidiaridad: Todo sistema es subsidiario, en su delimitación y en sus aportes de otros sistemas en virtud de los cuáles actúa y que forman su entorno.

b. Interaccion: Todos los sistemas que forman la empresa están mutuamente relacionados en su comportamiento.

c. Determinismo: Todo fenómeno que actúa en, o a través de los sistemas es el resultado de causas definidas y contrastables.

d. Equifinalidad: El sistema debe estar diseñado de forma que pueda alcanzar un mismo objetivo a través de medios y acciones diferentes entre sí.

\section{CARACTERÍSTICAS}

a. Estabilidad: El sistema debe ser capaz de mantenerse en funcionamiento eficaz, frente a las acciones de los factores externos al mismo.

b. Adaptabilidad: El sistema debe ser capaz de evolucionar dinámicamente con arreglo a su entorno, de manera que atraviese diferentes estados conservando su eficacia y su orientación al objetivo que constituye su finalidad.

c. Eficiencia: El sistema debe atender a su objetivo con economía de medios, poniendo en juego procesos que le permiten ser adaptable y equilibrado.

d. Sinergia: Su capacidad de actuación debe ser superior a las de sus componentes sumados individualmente.

\section{HACIA UNA PROPUESTA}

\section{Concepto de Congruencia}

En la actualidad se puede estudiar a las organizaciones bajo el concepto de modelo de congruencia del comportamiento organizacional. Es así que los componentes de toda organización coexisten en varios estados de equilibrio y consistencia, lo que se denomina ajuste o congruencia, para ello la interdependencia. Asimismo, actúan cuatro elementos fundamentales:

a. Insumos: entorno, recursos, e historia.

b. Estrategia: visión particular de cómo la organización se interrelacionará con el entorno; que defi- ne el conjunto de decisiones explícitas sobre los mercados, ofertas, tecnología y las competencias distintivas.

c. Productividad: se refiere a cómo la organización produce, cómo se desempeña y su grado de eficiencia.

d. Los procesos de transformación: el trabajo y los procesos comerciales que convierten los recursos en ofertas.

Por otro lado están los cuatro elementos de toda organizaicón: el trabajo, la gente, los acuerdos formales de la organización y la organización informal. Finalmente la congruencia también la podemos definir como el grado en que las necesidades, demandas, metas, objetivos y estructuras de un componente son consistentes con los del otro. Como la medición del nivel de la eficacia con que se ajustan los pares de componentes.

\section{Grupos Estratégicos}

La selección de la estructura básica: grupos estratégicos. El agrupamiento estratégico es el paso más importante en el proceso de diseño. Establece el marco básico para el diseño de la organizaicón. El agrupamiento unifica algunas tareas, funciones o disciplinas, en tanto que divide otras, en esencia concetra la atención de la organizacón.

El agrupamiento estratégico supone dos preguntas relacionadas:

- ¿Cuál es la configuración básica de la organización?

- ¿Cómo se especializan o diferencian los componentes organizacionales?

La elección inicial del agrupamiento estratégico y la configuración del núcleo de la organización son consecuencias directas de la estrategia de la organización o de la unidad.

Agrupamiento estratégico: las configuraciones básicas:

a. Agrupamiento por actividad o procesos.

b. Agrupamiento por servicios o productos.

c. Agrupamiento por usuario, cliente o área geográfica.

Las conexiones estratégicas, permiten la coordinación del trabajo. La base para las decisiones sobre las conexiones estructurales (agrupamiento) está arraigada en el concepto de la interdependencia de las tareas.

Con relación a las conexiones estructurales estratégicas se presenta una gama de opciones, de és- 
tas se opta las que favorezcan los flujos de información, procedimientos y estructuras adecuados para atender la interdependecia relacionada con el trabajo. La forma más obvia de conexión estructural es la jerarquía.

El diseño a nivel de la organización. Para ello se debe tener presente la gama de arquitecturas a nivel de empresa que existen: en un extremo la empresa integrada (organizaciones de procesos / horizontal) y al otro extremo las unidades de negocios independientes (redes organizacionales). El área de diseño, y que debemos escoger se encuentra en algún punto entre la empresa integrada y la red organizacional.

Para dicha elección se tiene en cuenta los conceptos de enfoque y apalancamiento. El enfoque, implica que una empresa consiste en una secuencia bá: ira de procesos que transforman las tecnologías (.). רfertas que se llevan al mercado. Esta operacinn yuc parte del fondo de la organización hacia el frente incluye: concebir, diseñar, desarrollar, fabricar, vender, distribuir y dar asitencia a una oferta.

El apalancamiento implica la capacidad de derivar ventajas de escala de los recursos comunes compartidos por la organización. Ello se encuentra en las áreas comunes de diseño, tecnologías básicas, capacidad de producción, redes de distribución o actividades relacionadas con clientes. Es así que surgen una serie de posibilidades: frente apalancado, fondo apalancado, medio apalancado, frente y fondo apalancado, unidades microempresariales.

\section{Diseño a nivel operativo}

Luego del diseño a nivel de toda la organización tenemos el diseño a nivel operativo, que implica tomar en cuenta los diseños tradicionales, los por procesos y lo más recomendado que son los diseños STAD Sistemas de Trabajo de Alto Desempeño parten de los principios socio técnicos iniciales:
a. Reglas mínimas.
b. Control de variaciones.
c. Multihabilidades.
d. Ubicación de límites.
e. Flujo de información.

Con la aplicación de los diseños socio técnicos surgieron dos ideas:

a. Que había una enorme fuerza en los equipos de trabajo autónomos.

b. Que en los años 60 y 70 el trabajo socio técnico se había llevado a cabo con un enfoque interno, pero que lo más eficaz emanaba de una perspectiva externa o de sistemas abiertos. Que se orientaban hacia los clientes, proveedores y competidores.
Esta experiencia se ha aplicado luego a los procesos de fabriación completos con éxito.Los conceptos esenciales que abarca este nuevo diseño son:

a. Selección de empleados.

b. Diseño de la planta física.

c. Diseño de los puestos.

d. Sistemas de pago.

e. Estructura organizacional.

f. Capacitación.

g. Filosofía gerencial.

Es clave para la aplicación de los STAD que cada organización debe diseñar su propia versión consistente con su trabajo y cultura. Sin embargo hay 10 principios que se consideran fundamentales para lograr ello:

a. El diseño empieza con un enfoque externo en las necesidades del cliente y de ahí parte hacia el interior para establecer las configuraciones organizacionales y los procesos de trabajo apropiados.

b. El trabajo está diseñado en torno de unidades autodirigidas, interralacionadas de manera flexible y encargadas de generar productos o procesos completos.

c. El trabajo debe estar orientado por una dirección clara, metas explícitas y una comprensión cabal de las necesidades de producción y las mediciones de desempeño.

d. Las variaciones deben detectarse y controlarse en su origen, en lugar de inspeccionarlas fuera de la unidad de trabajo.

e. Los sistemas sociales y técnicos se relacionan estrechamente; cada uno de ellos está diseñado con los otros en mente para producir el desempeño óptimo.

f. El flujo sin trabas de toda la información pertinente hacia la gente que la necesita es crucial para el diseño de los STAD.

g. Los puestos más interesantes y compartidos aumentan la motivación de los individuos y dan mayor flexibilidad a la organización para asignar trabajo y resolver problemas.

h. Las prácticas de recursos humanos deben complementar y fortalecer la delegación de autoridad en los equipos e individuos.

i. La estructura, cultura y procesos gerenciales deben aceptar y apoyar el diseño de los STAD.

j. La organización y sus unidades de trabajo deben tener la capaidad de reconfigurarse para enfrentar las condiciones cambiantes de la competencia. 


\section{CONCLUSIONES}

La organización debe considerarse como un sistema altamente integrado, cuyo desempeño esté determinado por el grado de disuasión entre sus principales componentes.

La gama de arquitecturas a nivel de empresas: integradas, horizontal y en redes, que se deben escoger, implica una secuencia de procesos que transforman la tecnología en ofertas que se llevan al mercado.

\section{BIBLIOGRAFÍA}

1. Kendall, K.E. y Kendall, J.E. 1997. Análisis y Diseño de Sistemas. Edit.Prentice Hall Hispanoamericana S.A. México.

2. Murdick, R.G. 1990. Sistemas de formulación administrativa. Edit. Prentice Hall Hispanoamericana S.A. México

3. Whitten, J.L. y Bentley, L.D. 1996. Análisis y Diseño de Sistemas de Información. Edit. Mc Graw Hill. Colombia. 